\title{
Functions in Basic Formal Ontology
}

\author{
Andrew D. Spear ${ }^{\mathrm{a}, *}$, Werner Ceusters ${ }^{\mathrm{b}}$ and Barry Smith ${ }^{\mathrm{c}}$ \\ a Philosophy Department, Grand Valley State University, 1 Campus Drive, Allendale, MI 49401, USA \\ Tel.: +1 616331 2847; Fax: +1616331 2601; E-mail: speara@gvsu.edu \\ ${ }^{\mathrm{b}}$ Departments of Biomedical Informatics and Psychiatry, University at Buffalo, 77 Goodell street, \\ Buffalo, NY 14203, USA \\ E-mail: ceusters@buffalo.edu \\ ${ }^{\mathrm{c}}$ Department of Philosophy, University at Buffalo, 135 Park Hall, Buffalo, NY 14260, USA \\ E-mail:phismith@buffalo.edu
}

\begin{abstract}
The notion of function is indispensable to our understanding of distinctions such as that between being broken and being in working order (for artifacts) and between being diseased and being healthy (for organisms). A clear account of the ontology of functions and functioning is thus an important desideratum for any top-level ontology intended for application to domains such as engineering or medicine. The benefit of using top-level ontologies in applied ontology can only be realized when each of the categories identified and defined by a top-level ontology is integrated with the others in a coherent fashion. Basic Formal Ontology (BFO) has from the beginning included function as one of its categories, exploiting a version of the etiological account of function that is framed at a level of generality sufficient to accommodate both biological and artifactual functions. This account has been subjected to a series of criticisms and refinements. We here articulate BFO's account of function, provide some reasons for favoring it over competing views, and defend it against objections.
\end{abstract}

Keywords: Function, disposition, Basic Formal Ontology, biological function, artifacts, malfunction

Accepted by: Pawel Garbacz

\section{Function and ontology}

It seems clear that any ontology intended for use in the domain of biology will eventually need to include the category of function, while any ontology intended for use in the domain of biomedicine including for example surgery or radiography - will need to include an account that can handle both biological and artifactual functions. Functions are ontologically challenging in that they are generally taken to involve a normative element: for a thing to have a function is for it to have something it is supposed to do and that it can do more or less well. There is a large and still growing literature on function (Buller, 1998; Wouters, 2005; Krohs and Kroes, 2009) and the topic has a long history documented for example by Gilson (1984) and Leunissen (2010). When approaching a complex issue of this sort it is therefore helpful to approach it with some basic ontological commitments already in place, and viewing the issue of function through the lens of an already developed and widely used top-level ontology is one effective way of doing this. This essay may be read, in part, as an illustration of how a formal ontology can constrain and assist in the development of an elucidation of a complex ontological phenomenon such as function. In the following we introduce the core commitments of Basic Formal Ontology (BFO) and

\footnotetext{
*Corresponding author. Tel.: +1 616331 2847; Fax: +1 616331 2601; E-mail: speara@gvsu.edu.
} 
consider in this light some influential accounts of function taken from the recent literature. In the final section of the paper we defend the BFO elucidation of function against a number of objections, finishing with those recently lodged by Röhl and Jansen (2014) and to some extent reiterated by Marc Artiga in his introduction to this special issue.

\section{Basic Formal Ontology}

BFO is a top-level ontology initially developed for use in scientific domains such as biomedicine. ${ }^{1}$ BFO sees reality in terms of a top-level division of all particular entities (individuals) into the two disjoint categories of continuant and occurrent. Continuant entities include objects, attributes, and locations and are contrasted with occurrent entities, which include processes and temporal regions. Processes happen in time and so have temporal parts (for example, the beginning, middle, and end of a cricket match). Continuants, in contrast, exist in full at any time at which they exist at all. It is accordingly not the organism or cricket ball that has temporal parts but rather its associated life or history. The is_a hierarchies in Figs 1 and 2 summarize the continuant and occurrent halves of BFO, respectively.

In addition to the distinction at the level of particulars between continuants and occurrents, BFO presupposes also a fundamental division between universals and particulars. The former are repeatable features of reality, typically instantiated in multiple particulars. The latter are non-repeatable entities that instantiate universals but are not themselves instantiated by or in anything. BFO, in effect, is an attempt to capture the most general universals - those categories of entities which are instantiated in all domains of reality - on the basis of the assumption that universals at lower levels will be represented in domain ontologies created through downward population from BFO. According to the methodology of

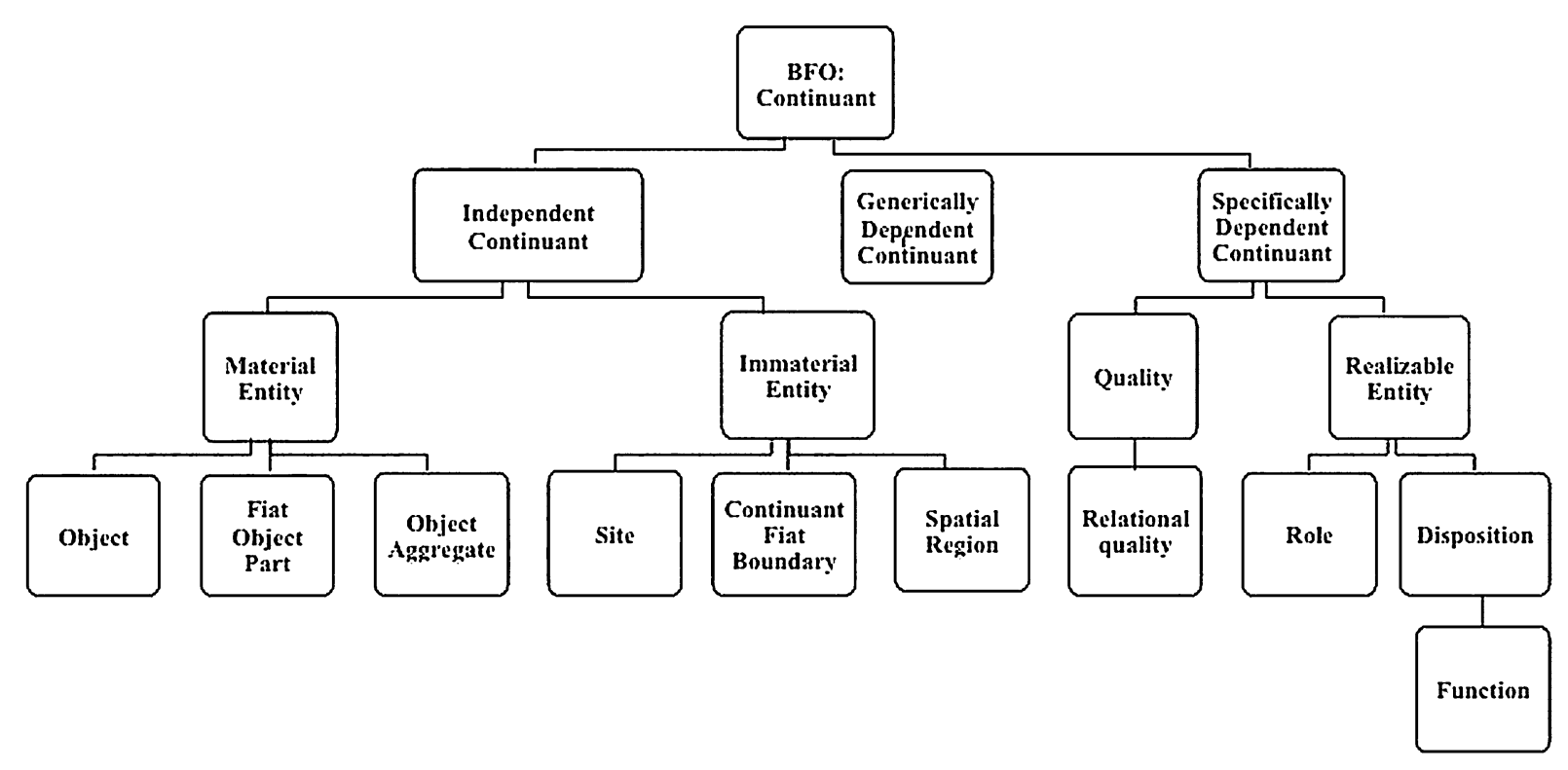

Fig. 1. Continuant universals in Basic Formal Ontology.

\footnotetext{
${ }^{1}$ The BFO 2.0 reference document (Smith et al., 2015) is available at https://github.com/BFO-ontology/BFO, accessed May 9, 2016. For a comprehensive introduction to Basic Formal Ontology and the approach to ontology on which it is based, see Arp et al. (2015). For a list of BFO users see http://ifomis.uni-saarland.de/bfo/users, accessed December 10, 2015.
} 


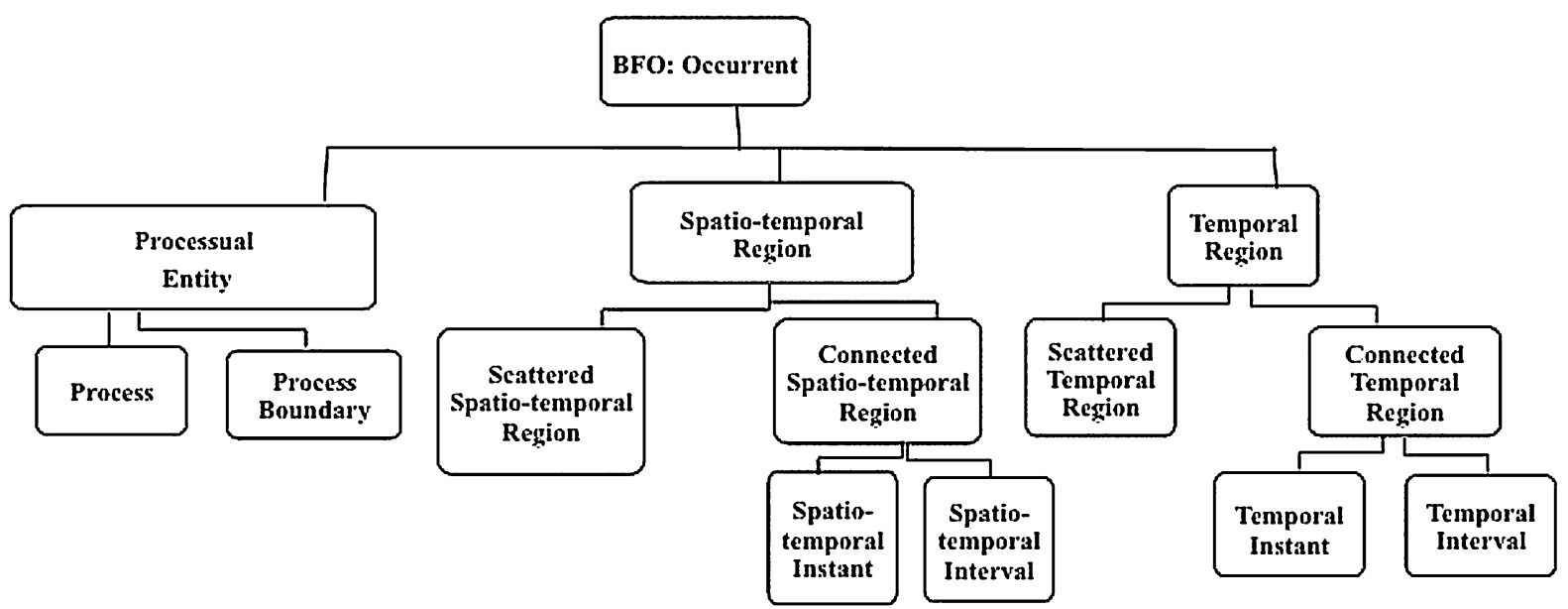

Fig. 2. Occurrent universals in Basic Formal Ontology.

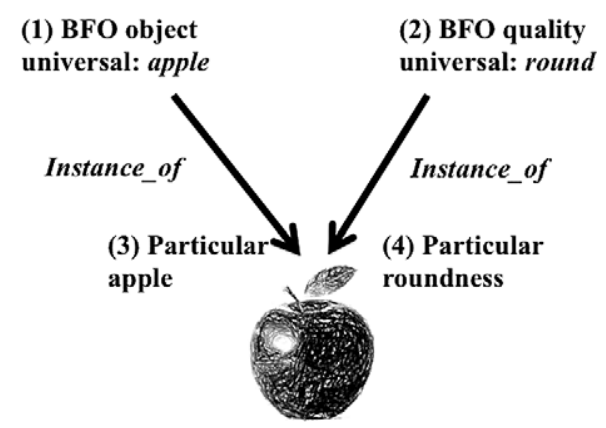

Fig. 3. Instances and universals together making it true that a certain apple is round.

ontological realism underlying BFO, general statements, such as mammals have hearts, electrons have negative charge, salt is soluble in water, or perspiring functions to cool the body - despite being defined in terms of particulars - are true or false in virtue of the existence of corresponding universals in reality. The universal heart, for example, is instantiated in each individual heart; the universal pumping in each individual process of pumping.

According to BFO, the truth of there is a round apple implies the existence of (at least) four distinct entities: the universal apple in the BFO category object, the universal roundness in the BFO category quality, an individual apple, and a particular instance of roundness inhering in the apple (see Fig. 3.)

BFO thus anticipates the four-category ontology defended by E. J. Lowe $(2006)^{2}$ but extends the latter to include universals and instances not only of dependent and independent continuants (roughly: objects and properties) but also of occurrents (Smith, 2005).

For our purposes here it is the dependent continuant BFO categories of quality, role, disposition, and function that are of most importance. On the ontological realist view, instances of universals in each of these categories exist in reality and are studied by science. Data about molecular functions, for example, are captured by the annotations created by biocurators using terms from the Gene Ontology Molecular

\footnotetext{
${ }^{2}$ See also Díaz Herrera (2006) and Smith (2012). Both BFO and Lowe, of course, belong to a long tradition, currently discussed for example under the heading 'Aristotle's ontological square'.
} 
Function Ontology (Ashburner et al., 2000). We will first consider the intuitive notion of function as it relates to accounts found in current literature. We will then introduce the BFO elucidation of function in the context of these accounts.

\section{Function}

We take it to be characteristic of what it is to have a function that for an object to have a function does not imply that it is realizing this function at every moment in which it exists, or indeed at any moment (consider a knife that is never used; or a sperm that never successfully fuses with an ovum). Functions are certainly intimately related to the processes that realize them, their functionings. But they are not identical therewith, and they are not existentially dependent thereon.

Second, the function of a thing is, approximately, what it is supposed to do. This typically involves both what is popularly called a goal or end of some sort and a way of achieving that end. A thing is functioning well when it does what it is supposed to do reliably or effectively and in the, for it, appropriate way. A thing is malfunctioning when it does what it is supposed to do poorly or not at all. The function of the heart is to pump blood. When this function is realized - when the heart is pumping then blood flows through the circulatory system. This is a consequence of the realization of the heart's function. A healthy or well-functioning heart does this well. An unhealthy or malfunctioning heart does this poorly. Similarly, the purpose of a pair of sunglasses is to shield the eyes from the sun or other source of bright light while still enabling vision. A good pair of sunglasses does this well. A defective or bad pair of sunglasses does it poorly (either by failing to shield the eyes from the light source or by obstructing the vision of the wearer). Talk of functions is thus in some sense teleological (involves reference to goals or ends or purposes) and normative (reference to functions serves as the basis of certain sorts of judgments of good and bad, better and worse).

Central tasks in elucidating the notion of function are, then, to account for the independence of functions from their functionings, and to account for the sense in which these functions are normative for the bearers that possess or instantiate them.

\subsection{Etiological and other accounts of function}

While there is a large array of different accounts of function in the literature, ${ }^{3}$ we distinguish here between three major positions:

- the etiological view (Millikan, 1984; Buller, 1999),

- the so-called "systemic" or "causal contribution" view (Wouters, 2005, pp. 135-136; Röhl and Jansen, 2014, pp. 6-7),

- "the life chances approach" (Bigelow and Pargetter, 1987; Wouters, 2005). ${ }^{4}$

Artiga (2011, p. 110) identifies four major desiderata that an account of function should try to satisfy. Reformulated slightly for the sake of terminological consistency they read as follows:

\footnotetext{
${ }^{3}$ See the surveys provided by Wouters (2005) and by Röhl and Jansen (2014).

${ }^{4}$ Note that all of these are realist views: they hold that functions exist, that they are ingredients of being. We do not here address those accounts - maintained for example by Searle (1995) - according to which function talk is a mere façon de parler about other things and thus in principle eliminable.
} 
- TELEOLOGY: reference to the function of an entity should play an important role in explaining why the entity exists.

- NORMATIVITY: the function of an entity determines a criterion against which the activity of the entity can be normatively evaluated, which means: it can be evaluated as better or worse relative to some norm relating to its exercise of the function.

- EPIPHENOMENALISM: the function of an entity is determined by the entity's current performance. ${ }^{5}$

- ACCIDENT: An entity's function is appropriately distinguished from its accidental effects (called "side-effects" in what follows).

Artiga also presents a strong case for the thesis that any account of function that satisfies the criteria of TELEOLOGY, NORMATIVITY, and ACCIDENT will likely fail to satisfy EPIPHENOMENALISM. In addition to the above desiderata, two additional criteria that will be of relevance below are discussed by Röhl and Jansen (2014), these are:

- SUPPORT: the function of a thing should be, in some sense, supported by (be a consequence of) its physical structure, not merely of historical or cultural facts. ${ }^{6}$

- MALFUNCTIONING requires of such a theory that it does justice to the phenomenon of malfunctioning, including the case where a thing manifests total failure to perform its function.

Röhl and Jansen recognize that there is a prima facie conflict between NORMATIVITY and SUPPORT similar in nature to the conflict between the four criteria identified by Artiga. It is characteristic of normative statements that they can be true even when the world fails to conform to them. Thus "John ought not to kill" does not imply "John does not kill". Similarly, for functional language, "John's heart is supposed to pump blood through his body" does not imply "John's heart does indeed pump blood through his body". Whatever functions are, there can be true statements about them (such as "the function of the heart is to pump blood") even when no actually existing thing does what those true statements purport (imagine a world in which all hearts have momentarily ceased functioning). SUPPORT, on the other hand, requires that functions be grounded in the physical structures of their bearers. According to this criterion, functions are not epiphenomenal add-ons or mere results of cultural context or reflections of widespread belief. That a thing has a function is supposed to follow, at least in part, from its physical structure. This implies that for a thing to have a function of a certain kind it must at the same time have a physical structure of a certain kind.

An account of function can thus be judged by how well it satisfies the desiderata just introduced, where it is probably the case that no account will satisfy them all. The BFO account of function to be introduced in more detail below is an etiological or history of selection account in the spirit of those offered by Millikan (1984) and surveyed by Buller (1998). For BFO, as we shall see, a function is a disposition of its bearer that (1) exists in virtue of the bearer's physical makeup where (2) this bearer came into existence as a result of a historical process of a certain sort (evolutionary selection for biological entities and intentional design or selection for artifacts). Our position is that the etiological view embraced by BFO fairs better, with regard to the desiderata distinguished by Artiga and by Röhl and Jansen, than do the other mentioned accounts.

\footnotetext{
${ }^{5}$ In Artiga's original formulation: "The trait's function is determined by the trait's current performances." Artiga does not provide a definition of what he means by 'trait' or by 'current'.

${ }^{6}$ This criterion is also discussed by Smith et al. (2015) under the heading of 'material basis'.
} 


\subsection{The systemic/causal contribution account}

Consider first the systemic or causal contribution accounts according to which the function of an entity has to do with the contribution that it makes to bringing about a state or behavior of some system or context of which it is a part (Cummins, 1975; Mossio et al., 2009). Both artifacts and biological entities are complex systems whose parts play certain roles in sustaining them and guiding their behavior. It seems, therefore, that a view along these lines is attractive. Further, the view seems to satisfy the EPIPHENOMENALISM constraint rather easily as it is built into the view that how the relevant part of the system is currently performing determines what its function is. However, such views do not do so well by the other three desiderata. Concerning TELEOLOGY: just because an entity plays a causal role in a system does not mean that its doing so explains why that entity exists or continues to exist. The right combination of sunlight and moisture in the air can produce a rainbow; but playing this causal role does not explain why sunlight or moisture exist. Concerning NORMATIVITY: the fact that some factor plays a causal role in a system does not imply that it is supposed to do so or that it does so more or less well. A heart in atrial fibrillation is making a causal contribution to the overall state of the system of which it is a part, but it would be a mistake to identify its quivering heartbeat as a function of the heart. Such a heart is precisely malfunctioning, and it is not clear how the causal contribution account can do justice to this fact.

Such accounts face for similar reasons a difficulty in distinguishing between the side effects produced by an entity functioning within a system and the functioning of that same entity. Insofar as both make causal contributions to some state of the system, both would seem to count as functions of the entity in question thus contravening the ACCIDENT desideratum. To see this, consider the version of the systemic or causal approach defended by Mizoguchi et al. (2012). This defines the function of an entity $\mathrm{A}$ in terms of the contribution A makes in the relevant systemic context $\mathrm{C}$. For $\mathrm{A}$ to have a function in $\mathrm{C}$, A must be a component of some subsystem of $\mathrm{C}$ and some behavior of A must play the functional role determined by $\mathrm{C}$. This definition of the function of $\mathrm{A}$ will work well so long as it is clear what defines the goal of the systemic context $\mathrm{C}$ to which A's functioning contributes. However, to determine what fixes this goal they must appeal to something external to the systemic context. In the case of artifacts, they appeal to the intentions of the designer of the system. In the case of biological entities it seems that they will be forced to draw on one or other of the extant accounts of biological function.

\subsection{The life chances view: Bigelow and Pargetter}

Bigelow and Pargetter (1987) offer a view of biological function that takes its inspiration from evolutionary biology but avoids considerations involving the selection history of organisms and their parts in favor of the possession by an organism or part of an organism of a "survival-enhancing propensity" in the present. As they put it, "Something has a (biological) function just when it confers a survival-enhancing propensity on a creature that possesses it" (Bigelow and Pargetter, 1987, p. 108). ${ }^{7}$ As Wouters, who calls this the "life chances view", puts it:

it is the function of the heart to pump blood because pumping blood is an effect of the heart and the life chances of organisms that have hearts are better if their heart pumps blood than if it does not (Wouters, 2005, p. 139).

\footnotetext{
${ }^{7}$ This account comes with provisos, the central one being that what counts as a survival-enhancing propensity for an organism is to be determined relative to that creature's "natural habitat", the one that is usual or normal for it.
} 
Views of this sort avoid the appeal to a history of selection used by etiological theories and distinguish themselves from systemic approaches by introducing survival (sometimes also reproductive success) into the determination of a thing's function. Their view does well in relation to the criterion of EPIPHENOMENALISM, since an organism's functions are explained in terms of its current propensities. It also satisfies ACCIDENT by conceiving functional (as opposed to accidental) effects as those that are survival-enhancing. However, it faces problems regarding TELEOLOGY and MALFUNCTION. First, it is not clear how an organism or organ having a survival-enhancing propensity in the present serves to explain the existence of that very propensity. On the view of Bigelow and Pargetter, an entity that has just come into existence and happens to have a survival-enhancing propensity for the organism would count as having a function, yet its having this function could not be part of the explanation for why it exists. Second, the view does little to distinguish, for example with regard to a given organ, what counts as functioning and malfunctioning, and so does little to satisfy NORMATIVITY. A heart that pumps blood surely confers a survival-enhancing propensity on an organism that would not be possessed by an organism whose heart did not pump blood. Thus, a heart that pumps blood counts as having the function to pump blood. But what about a malfunctioning heart? Such a heart may well confer a counterpropensity to survival on the organism that is its host, and thus not have a function. Yet this seems incorrect. The malfunctioning heart is supposed to pump blood and is failing to do so. We describe it as malfunctioning precisely because it has or is at least supposed to have a function.

\subsection{Virtues of etiological accounts of function}

Etiological views of function determine what the function of a thing is in the present by appeal to the history of that thing or of things like it. In the case of biological functions, the current function or functions of a thing will be determined by the evolutionary history of that thing, specifically by which characteristics of that thing played a role in the evolutionary success of ancestors possessing similar characteristics. In the case of artifacts, the current function of an artifact is determined by its history of intentional design or selection by some agent. Thus an etiological account is, by contrast with both systemic and life chances accounts, able to satisfy at least three of Artiga's four desiderata. It satisfies TELEOLOGY because it sees the function of for example an organ as determined by those traits of precursor anatomical structures that were selected for in ancestors of the organism. The function of the organ thus contributes to explaining, causally, its existence. Etiological views also handle NORMATIVITY and ACCIDENT. The heart is supposed to pump blood, not to make a thumping sound, because it is pumping blood that contributed to the survival of past members of the species who possessed hearts or precursor structures. ${ }^{8}$ The heart is supposed to pump blood because this is what was selected for in the history of organisms possessing hearts, and this is also why a heart that does not pump blood where it ought to do so, or that does not pump blood effectively, is malfunctioning.

The one desideratum that the etiological theory runs afoul of is EPIPHENOMENALISM, since it is not the current state of the organism or artifact that determines its functions, but rather the past history of selection. EPIPHENOMENALISM is however by far the weakest of Artiga's desiderata, since it clearly cannot deal with a host of cases where entities - both artifacts and biological continuants have functions but are not at any given time functioning. Artiga indeed - as is clear in his formulation

\footnotetext{
${ }^{8}$ It is of course possible that there is some selective advantage in pumping with a certain amount of noise, for instance because more noise might have made certain animals more likely to be heard by predators, less noise might have made their mothers think that they were dead while they were merely asleep, resulting in abandonment. Pumping blood outside the relevant noise thresholds would then be a case of malfunctioning.
} 
of ACCIDENT - does not seem to clearly distinguish between function (continuant) and functioning (occurrent). We hypothesize that his acceptance of EPIPHENOMENALISM flows from the fact that he is seeking to answer epistemological questions (how can we know or explain that a function exists?) rather than the ontological question (what is it for an entity to be a function?) which is of interest to us here.

It is for all of these reasons that we adopted the etiological account in the BFO elucidation of function. ${ }^{9}$ BFO retains the Bigelow and Pargetter thesis that a function is something like a propensity (for us: a disposition) that an entity has; but adds to this the view that the normative features of this propensity, its status as a function proper, are to be derived from it being the outcome of particular kinds of historical processes.

\section{Disposition, role, and function in BFO}

As can be seen in Fig. 1, dispositions, roles, and functions are all, from the BFO standpoint, specifically dependent realizable continuant entities. A continuant entity is dependent if, in order for it to exist, it must inhere_in some other entity, for example as a quality of roundness inheres in some round object. ${ }^{10}$ The temperature of a motor depends on - and so inheres in - the motor; and the fragility of a vase depends on - and so inheres in - the vase.

To see what BFO means by realizable, note that, as the previous examples illustrate, dependent entities divide into (at least) two significant kinds:

1. those that are completely exhibited by an object at all times at which they exist at all (such as temperature, height, diameter, thickness, mass, and shape),

2. those that are associated with a characteristic process of realization or manifestation - as in the case of fragility, elasticity, the ability to play tennis, the responsibility of a legal guardian, the duties of a policeman.

Dependent entities of the first type fall under the BFO category of quality; those of the second type are included under the category of realizable entities and are distinguished by the fact that they are features of an object that are only realized or become manifest under certain conditions. Realizable entities are therefore in a sense modal: it is only possible that they be realized. A vase is always fragile, but its disposition to break is only realized or made manifest when (or if) it is, for example, dropped or hit with a hammer. Dispositions, functions, and roles are all realizable features of their bearers, and thus they are all first class entities in the BFO sense. Committing to their existence provides us with a way of dealing with modality which does not require appeal to anything like modal logic or possible worlds.

Dispositions are the most familiar of these three types of realizable entity. Examples include the disposition of a vegetable to decay; the disposition of a block of ice to melt; the disposition of a portion of blood to coagulate; the disposition of a patient with a weakened immune system to contract infectious

\footnotetext{
${ }^{9}$ We use the term 'elucidation' here in reflection of the fact that the categories in a top-level ontology are generally so basic to our understanding of reality that it is difficult or impossible to provide definitions of them in the strict sense of the term. To provide elucidations of such categories means to describe the key features that entities falling within these categories have by providing examples and by specifying axioms that represent how these categories relate to others within the ontology (Arp et al., 2015, pp. 89, 135-136).

${ }^{10} \mathrm{BFO}$ also makes use of the distinction between generically dependent and specifically dependent continuant entities, but this distinction will not be important in what follows here.
} 
disease; the disposition of a portion of metal to conduct electricity. There is a vast philosophical literature on dispositions (also sometimes called 'powers') ranging over a number of significant metaphysical issues. ${ }^{11}$ While BFO is a realist ontology committed to taking such issues seriously wherever they might help to identify potential problems with the BFO framework, BFO's primary goal is to assist scientists and others in the development of practically useful, accurate, coherent, and interoperable domain ontologies by providing a starting point for downward population through the formulation of Aristotelian definitions (Arp et al., 2015, p. 69). As such, BFO incorporates ontological categories and associated elucidations that have proven relevant to the practical endeavors of information-driven scientists while, wherever possible, remaining neutral on more contentious questions of metaphysics.

Dispositions are described by reference to the types of processes which would realize them under certain conditions (Ellis and Liese, 1994). To be fragile is to break under these or those circumstances; to be soluble is to dissolve in liquid under these or those other circumstances. Each disposition will exist because of some physical quality or qualities of its bearer, but different quality patterns or arrangements may serve as this ground or basis under different circumstances or in different types of bearers. For example, the disposition of fragility might be instantiated in virtue of the different underlying molecular structures of a dry pine board, an ice sculpture, or the inflorescence of a rose.

Whether a disposition is realized will in many cases depend on the situation in which the object possessing it is located. Matches have in normal circumstances the disposition to be flammable, and this disposition will be founded on certain qualities of the matches. However, a match possessing the normal set of flammable-making qualities (of, predominantly, portions of phosphorus, sulfur, and potassium chlorate) will not be able to realize its disposition if it is located in an environment from which all oxygen has been removed. ${ }^{12}$

Changes in the environment of an object may result in temporary or even complete loss of dispositions, but only if they also bring about physical changes in the object for instance of the sort which occur when a match is inserted into water (Mumford, 1996). The environment, from the BFO perspective, cannot bring about these effects alone, since the dispositions that an object has are determined by its physical structure (a pattern of qualities in BFO terms).

Accordingly, the current BFO 2.0 elucidation of disposition reads as follows:

$d$ is a disposition means:

$d$ is a realizable entity,

$\& d$ 's bearer is some material entity,

$\& d$ is such that, if it ceases to exist, then its bearer is physically changed,

\footnotetext{
${ }^{11}$ Examples of such issues include:
}

- Is the correct analysis of dispositions to be achieved through logical analysis of sentences such as "this vase is fragile" or in terms of conditionals along the lines of: "this vase is fragile if and only if were it to be struck with a hard object it would crack or shatter"? (Lewis, 1997; Fara, 2005).

- If we refer to a patient with a weakened immune system as having an enhanced disposition to contract some disease, are we referring to just one disposition (to contract disease), or to what may be a large number of distinct dispositions (to contract influenza, to contract pneumonia, and so on)? And as the disposition (for example) to contract the flu becomes stronger or weaker is there then some single disposition that is undergoing a series of changes or is it rather a series of dispositions (each weaker or stronger than the next, and each giving way to its successor) as the patient's condition evolves with time? (Lowe, 2010).

- Do dispositions ground laws of nature, or do they themselves require some further categorical (which is to say nondispositional) ground or basis, for example in molecular structures or patterns? (Handfield, 2009).

${ }^{12}$ Realizability may also be affected by the presence of blocking dispositions (Goldfain et al., 2011). 
$\& d$ 's realization occurs when and because this bearer is in some special physical circumstances, $\&$ this realization occurs in virtue of the bearer's physical make-up.

Roles, by contrast with dispositions, do not follow from fundamental features of the physical structure of their bearers. Rather, they have to do with the natural or social context in which their bearers are located. Examples include the role of a person to be a doorman, the role of a chemical compound to be an analyte in an experiment, the role of a drug in the treatment of a disease, the role of a certain piece of fruit as food for an animal, the role of a patient's family member as defined by a certain hospital administration form, and so on. What is crucial for understanding roles is that they are realizable features that an object can have, but that it need not have. Thus roles are always optional. For example, when a piece of granite is used to mark the property line between two houses, then it has the role of property line marker; but if the same piece of granite is used in a museum as an example of a property marker then it has instead the role of a historical display object.

The current BFO 2.0 elucidation of role reflects these points:

$r$ is a role means:

$r$ is a realizable entity,

$\& r$ exists because there is some single bearer that is in some special physical, social, or institutional set of circumstances in which this bearer does not have to be,

$\& r$ is not such that, if it ceases to exist, then the physical make-up of the bearer is thereby changed (Smith et al., 2015).

\subsection{BFO function}

The BFO ontology of realizable entities does not claim to be complete. Currently it divides realizable entities into the two disjoint subcategories of disposition and role; but it is possible that further subcategories will be recognized in the future in order to address as yet unidentified needs (for example arising from new scientific discoveries or from applications of BFO to new sorts of domains). ${ }^{13}$ Already now, however, BFO recognizes function as a subcategory of disposition, and thus as a third realizable entity category. Examples under this heading are: the function of a liver to store glycogen, the function of a birth canal to enable transport, the function of an eye to see, the function of a water pump to move water, the function of a mitochondrion to produce ATP, and so on.

Note that these are all examples of objects (or, more generally, material entities) having functions of one or other sort. There are some who talk of entities in other categories, for example of certain types of processes, as having functions. Certainly, by definition, processes do not have functions in the BFO sense of this term. But BFO makes no claim to completeness, and thus it leaves open whether there are entities properly to be conceived of as functions of processes which might form an additional category deserving to be recognized by BFO. Favored examples of functional processes - for example the cycle of green-red or of green-amber-red illuminations of traffic lights, or the process of blinking when exposed to bright lights, or the process of blood flowing through the body to bring oxygen to its cells - are all, as far as we have been able to ascertain, capable of being treated as examples of functions of material entities in the BFO sense (of traffic light installations, of the upper eyelid musculature, and of the circulatory system, respectively) (Smith et al., 2003).

\footnotetext{
${ }^{13}$ On the open-endedness of BFO's ontology design process, and also on its commitment to fallibilism and, more generally, to the importance of responding to developments in science, see the discussion by Arp et al. (2015, Chapter 3).
} 


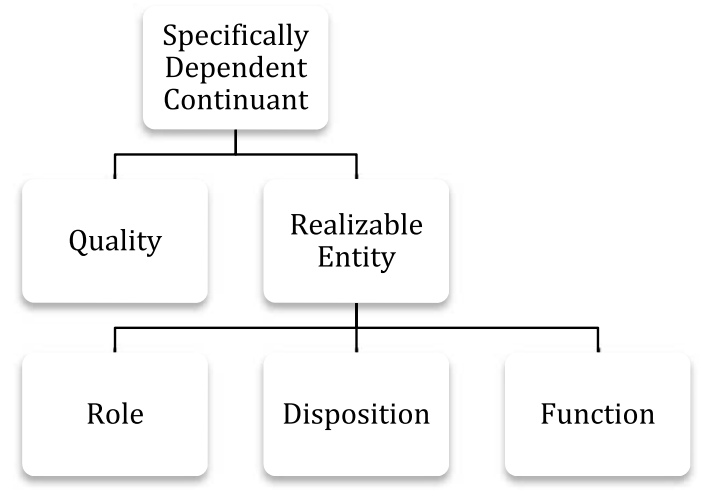

Fig. 4. Specifically dependent continuants in BFO 1.1.1.

\subsubsection{Function in BFO 1.1.1 and BFO 2.0}

BFO by now has some hundreds of users, including not only many biological and biomedical research consortia but also major government agencies in defense and security. ${ }^{14}$ To accommodate the needs of these users we have sought to ensure that changes to BFO are carried out only very infrequently, and that when these changes do occur they are as non-disruptive as possible to the ontology as a whole. In all, three versions have been approved since the original BFO 1.0: versions 1.1, 1.1.1 and 2.0 respectively. We here consider only the two most recent versions, since their different treatments of function have a direct impact on our arguments here.

In BFO 1.1.1, first of all, function, role, and disposition are treated as sibling categories on the same level, with realizable entity as their common parent, as in Fig. 4 above.

The elucidation of function provided by Spear (2006) and encoded in BFO version 1.1.1 reads as follows:

[a function is] a realizable entity the manifestation of which is an essentially end-directed activity of a continuant entity in virtue of that continuant entity being a specific kind of entity in the kind or kinds of contexts that it is made for (Spear, 2006).

This elucidation captures some important features of the notion of function. In particular, it includes the idea that the function of a thing is directly connected to what kind of thing its bearer is. However, it leaves open exactly how the function of a thing is related to its other qualities and to its parts. This elucidation also leaves unspecified just how the function of a thing is determined. Of all of the features of a thing, which are the ones that are to count as functions? Hanging in the balance here was also the need to clearly distinguish between functions on the one hand and roles on the other. Functions are supposed to be essential or integral to their bearers in a way that roles are not. But what grounds this difference?

It was primarily in order to respond to questions of this sort that the elucidation of function was modified in BFO 2.0 to:

$f$ is a function means:

$f$ is a disposition,

$\& f$ exists in virtue of its bearer's physical make-up,

\footnotetext{
${ }^{14}$ For a list of the (currently 71) scientific ontologies that reuse the BFO elucidation of function, go to: http://bioportal. bioontology.org/search?utf8=\%E2\%9C\%93\&query=function\&commit=Search and scroll down to BFO:Function.
} 
$\&$ this physical make-up is something that this bearer possesses because it came into being, either through evolution (in the case of natural biological entities) or through intentional design (in the case of artifacts), in order to realize processes of a certain sort (Smith et al., 2015).

The category of functions in BFO 2.0, accordingly, is identified as a subcategory of dispositions. Specifically, functions are dispositions whose bearers have specific histories of intentional design or evolution. Since dispositions are themselves a reflection of the physical structure of their bearers, it follows that functions, too, reflect this same physical structure. This means in turn that a loss of function or a failure of functioning on the part of the bearer - with certain exceptions which we will identify in what follows - implies some change in this bearer's physical structure. Recognizing that functions are types of dispositions serves also to differentiate them from roles, since the latter can be gained and lost without any change in the physical features of the relevant bearer. A piece of granite can start and stop playing the role of a property marker and a human being can start and stop having the role of being a bar-certified lawyer without undergoing any change in physical structure. The most obvious way for a heart, by contrast, to lose its function is for the heart to undergo some physical change of this sort.

\subsubsection{Functions as dispositions}

In addition to the above, five further considerations speak in favor of the view that functions are, ontologically speaking, dispositions.

First, while functions are most identifiable when they are being realized in processes of various sorts, that is in functionings, it is a normal occurrence for objects that have a function not to realize that function at all. Many (indeed almost all) spermatozoa never fertilize an ovum, yet it remains true that this is their function. A plastic fork may never actually be used for eating (it may be destroyed or thrown away before such use), yet its function is to serve the lifting of food all the same.

Second, otherwise indistinguishable processes can be functionings in some cases and not in others. Examples would include a cooling process, a process of wind-propelled flight, or a cutting process. If the cooling process is realized as a result of the bodily dispositions responsible for perspiration, then it is a functioning. If it is brought about as a result of the death of the organism, then it is not. The difference is not in the type of process itself.

Third, that an entity has a function is supposed to play a role in explaining the existence of that entity, or at least of some parts of that entity (this is Artiga's TELEOLOGY desideratum). Assuming a view of explanation that requires a causal component, it follows that dispositions are a good candidate continuant entity to do this explaining, because they are centrally involved in the physical makeup and causal behavior of the entities that possess them.

Fourth, we think that functions, when they exist, are both subject to causal influences and themselves have a causal impact on the world via their manifestations, and dispositions satisfy both of these conditions.

Fifth, and finally, the function of a thing is supposed to be in some sense fundamental or central to that thing or to things of that kind. By identifying functions with dispositions we are tying the notion of a thing's having a function directly to that thing's physical structure. On the assumption that some aspects of a thing's physical structure are fundamental to it, we are then tying functions to fundamental features of their bearers by identifying those functions with (certain) dispositions. 


\subsubsection{Functions as dispositions with an etiology}

Functions in BFO 2.0 are dispositions which are the result of a certain kind of historical development. ${ }^{15}$ In the biological case, a part or system in an organism has a function now if having that part or system has contributed in the past to the survival and reproductive success of members (or ancestors of members) of the species to which the organism belongs. ${ }^{16}$ It is not the physical structure and disposition alone that determines an entity's biological function, but rather the way in which physical structures of the relevant type have affected the fitness of their bearers and of their precursor organisms. Thus, when we say that the function of the heart is to pump blood, we are saying this in virtue of our recognition of the fact that organisms whose hearts have done this in the past have for this reason enjoyed enhanced prospects of survival and reproductive success. In the artifact case, when we say that the function of a calculator is to carry out various kinds of mathematical operations we are saying this because we know that the item in question was intentionally designed and made by some intentional agent to do precisely that.

\section{Implications of the BFO elucidation of function}

In this section we develop some of the implications of the BFO elucidation of function with special attention to the ways in which changes in history can effect changes in function, and the persistence conditions of function instances, especially in cases of transplant (such as the relocation of a watch spring from one watch to another, or of a heart from one person to another).

\subsection{An example of evolutionary change}

The evolutionary view of biological function encoded in BFO implies that (i) physical structures that currently have no function might have precursor physical structures that had a function, and that (ii) a physical structure that currently has a function might have descendant physical structures of which this is no longer the case. Moreover, (iii) physical structures that currently have one function could, in the course of time, have descendant physical structures with additional or entirely different functions. ${ }^{17}$ As is well known, the processes of evolution are such that they often involve types of physical structure being repurposed to new ends in the course of time. It is then sometimes difficult to determine which processes are the relevant functional ones on the basis of current knowledge. ${ }^{18}$

To see how this works consider the following example. Currently, what we can call the glycogen system functions in the presence of certain enzymes to synthesize glycogen in amounts necessary to

\footnotetext{
${ }^{15}$ Note that 'history' is used in BFO in a technical sense: the history of a material entity is for BFO the sum of all processes occurring in the spatiotemporal region occupied by that entity over the course of its existence. Thus we use 'historical development' in what follows to avoid confusion with this usage.

${ }^{16}$ Millikan (1984) is a classic statement of the evolutionary view of biological function, while the introduction in Buller (1999) provides a helpful overview. On biological systems - for instance the cardiovascular system - as parts of organisms see Smith et al. (2003). Following the account of 'canonical anatomical structures' in Rosse and Mejino (2003), we may say that a biological function is a function which inheres in an entity that is (i) part of an organism and (ii) exists and has the physical structure it has as a result of the coordinated expression of that organism's structural genes.

${ }^{17}$ Some recent research suggests that the appendix may be an example of a biological structure with just such a history, having first functioned in digestive processes, then become vestigial (and so lost this function), and yet later taken on a function in immune responses to stomach illness (Laurin et al., 2011).

${ }^{18}$ Epistemological considerations of this sort seem to be a large part of the motivation behind Amundson and Lauder's (1994) criticism of etiological accounts of function. Our own view is that the first task in accounting for function is to elucidate correctly what functions are. This makes epistemological issues orthogonal to our main concern.
} 
maintain blood glucose levels (Berg et al., 2002). Suppose that through some mutation a different system develops that more successfully regulates the amount of glucose present in the blood. Imagine now that under these conditions some organisms in the population continue to synthesize glycogen in the old way, while some begin to take advantage of systems of the new type. The latter will then have a decreased risk of developing a glycogen storage disease and will thereby enjoy an evolutionary advantage. It is a consequence of our view that, in the initial generations after a mutation of this sort, even in those organisms that can avail themselves of both the old and the new and more efficient system, it will still be the old system that has the function of regulating blood glucose. In course of time, however, on the assumption that the new system proves evolutionarily advantageous, the new system will take over from the old, and it will then be the new system that will have this function. This is in spite of the fact that the physical structure of the original system, taken by itself, need not be changed in any way in the organisms which survive to realize the corresponding dispositions. This is also in spite of the fact that we have no way of knowing when, precisely, the switch occurs. Evolutionary changes are almost always such that it is only retrospectively - often after many generations - that we are able to assert that the change in question has occurred.

This example, which we take to illustrate a phenomenon that is relatively common in evolutionary history, shows the crucial role played by the historical element in the BFO 2.0 elucidation of function. Every function is a disposition, but for a disposition to be a function the relevant historical elements must be in place as well - and what these historical elements are may be determined only in the course of time, when we are able to see which of (for example) two alternative systems survives in a given species and which becomes vestigial. We will see below that similar considerations apply also to the case of artifactual functions.

\subsection{A potential dilemma for the view of functions as dispositions with a history}

The discussion above makes it necessary to amend one common way of characterizing BFO function according to which it is the case that, because functions are dispositions, the loss of a function always entails some physical change in its bearer. ${ }^{19}$ Certainly it is true that the loss of a disposition involves a change of this sort. But this will not always be so in case of the loss of a function. The retained old glycogen system still has the disposition to synthesize glycogen, but it is no longer the function of the corresponding physical structures.

It will still be true that in almost every case a bearer's losing a function is because of some physical change in the bearer: it is because a heart has been severely punctured that it no longer has the function to pump blood; it is because a fan heater has been crushed that it will no longer have the function to emit heat. However, as the glycogen synthesis example once again shows, it is possible for a given disposition of a given bearer to become or to cease to be a function of that bearer solely as a result of certain changes in the bearer's history.

We can now see how we can address an apparent dilemma in our approach, which might be formulated as follows:

An entity can acquire a function through mere selection.

An entity cannot acquire a disposition through mere selection.

How then it is possible that every function is a disposition?

Another formulation is:

\footnotetext{
${ }^{19}$ See, e.g. the discussion by Arp et al. (2015, pp. 102-103).
} 
An entity can acquire (or lose) a disposition only through some physical change in the entity.

An entity can acquire (or lose) a function without any physical change in the entity

How then is it possible that every function is a disposition?

\subsubsection{Responding to the dilemma}

The dilemma is resolved precisely through the BFO definition of a function as a disposition of a bearer with a specific kind of historical development. In the biological case the answer is that dispositions that were functions for an ancestor of a given organism (and so of its parts) can have ceased to be functions for the current organism as a result of the fact that ancestors still more recent in time were not evolutionarily successful as a result of possessing the dispositions in question. The glycogen example above is such a case, and it illustrates how the mechanisms of historical selection themselves might bring it about that an entity whose physical structure (and concomitant dispositions) remain unchanged from one generation to the next may nevertheless gain or lose a function that is (or is not) realized by the physical structure and dispositions in question.

The situation is trickier for artifacts. Whereas the gain or loss of biological function by a single physical structure will typically take place across successive generations of organisms belonging to the same species, it is possible for a natural object (such as a stick, a tree, or a cave) to be assigned a use by intentional agents merely as a result of their selecting the object for such use without any further change to its physical structure. ${ }^{20}$ Does such a selection result in the object having a function in just the way that ordinary artifacts that have been designed and manufactured do? To answer yes would be to imply that a smooth rock selected from the lakeshore and used as a paperweight would exemplify the exact same function universal as would a stone paperweight that had been intentionally designed and manufactured for this purpose.

But there is an alternative position, which is to maintain instead that cases of novel use or repurposing of an object do not amount to its acquiring a new function, but rather involve an already existing natural object or artifact taking on a new role in the technical BFO sense of this term discussed in Section 4 above. ${ }^{21}$ Here and in what follows we adopt this second option and so reject the idea that the mere finding and repurposing of an already existing entity amounts to that entity taking on a new artifactual function. $^{22}$

\subsection{Function instances and transplant cases}

We have been considering more specifically the conditions under which an instance of function can come into or go out of existence. In some artifactual cases, for example hammers or chairs, it is the whole object which is the bearer of the function. In other cases, however, and in particular in all biological

\footnotetext{
${ }^{20}$ Compare Dipert's view (1993), according to which the artifactuality of an entity rests not on physical qualities we can presently see but on features of the entity's deliberative history.

${ }^{21}$ Artifacts that have been intentionally designed and made for one purpose can be selected for use in some other way by intentional agents (such as an anvil that is repurposed for use as a doorstop or paperweight without undergoing any changes in its physical structure). When portions of a drug, manufactured to serve the function of curing disease A, are discovered to be effective in curing a second disease $\mathrm{B}$, then these portions play the curing disease $\mathrm{B}$ role. Portions of the same drug manufactured at a later stage with the intention of being used to cure disease B, in contrast, can properly be said to have the corresponding function.

${ }^{22}$ For further consideration of this view see Section 7.1 below and our response to Marc Artiga's introduction to this special issue of Applied Ontology appended to this essay. We are indebted to Artiga and to an anonymous reviewer for pressing us for clarity on this point. The position taken here is consistent with what is said about BFO function by Arp et al. (2015) and by Smith et al. (2015). It revises some claims made by Spear and Smith (2015).
} 
cases, we will require a distinction between the bearer of the function (such as a heart or lung) and the organism of which this bearer is a proper part. An important question, now, is whether, in the latter sort of case, an entity loses its function when it is removed from its circumcluding host, for example in a case of transplantation.

For BFO, functions are continuants. Thus, like other continuants, they persist through time. An independent continuant such as a mouse persists as the same (numerically identical) mouse from the beginning to the end of its existence, even while gaining and losing new parts and new qualities as it develops and grows. Dependent continuants, too, persist through time from the BFO perspective, and thus in particular so also do instances in the category function. As the mouse develops and grows so also, in the normal course of events (the course of events typical or canonical for instances of this species), does its heart, which initially grows larger and stronger until the mouse reaches its peak of cardiac fitness from where it then slowly descends to the point of cardiac arrest. Through all of this, the function of the mouse's heart remains identical, even though it is realized in different phases of the mouse's life through functionings (above all processes of pumping blood) which manifest characteristic differences in virtue of increasing and decreasing cardiac capacities.

A watch spring, similarly, has a single identical function - of storing mechanical energy to power a watch - from the beginning to the end of its existence, and this function remains in existence even when the watch is taken apart for repair, and even when the remainder of the watch is destroyed (since the spring retains exactly the dispositions it had when first created, as is shown by the fact that these dispositions are capable of being realized, for example, if the spring is transferred to a new housing). It is for this reason that, when we discover intact stone artifacts, some of them dating back millions of years, we can in very many cases not merely determine what functions they were initially designed to serve, but also exercise these same functions in actions that we ourselves perform.

And so too, we now argue, for the biological case. The function of the mouse's heart (this function of this mouse's heart) is preserved even when the heart is transplanted into another mouse. It would be preserved even were it to be transplanted from the mouse to some cardiac chamber that functions (perhaps for purposes of storage or of research) to preserve those physical features of the heart in virtue of which it has the disposition to pump. The cardiac chamber preserves, in such a case, not merely the disposition, but also the function of the particular heart in question.

\section{Objections to the BFO/etiological view}

In this section we respond to three objections to the BFO elucidation of function. We then consider in Section 7 a direct challenge to the BFO identification of functions with dispositions.

\subsection{Etiology and the epiphenomenalism desideratum}

A first objection to the BFO view is that, because we reject Artiga's criterion of EPIPHENOMENALISM, this means that we view functions as epiphenomenal. For BFO, however, functions are a special subtype of dispositions, and to say that functions are dispositions is not to say that they are causally inert. Indeed quite the contrary: dispositions and their realizations are paradigmatically caught up in causal relations in nature, so that any function will itself be caught up in relations of being caused and of causing other things. We thus need to distinguish two ways in which the term "epiphenomenal" might be understood when applied to functions: as asserting (1) that for an entity to have a certain function it is not necessary that there be current realizations of this function; or (2) that the function has no causal impact 
in nature whatsoever. We accept (1) without hesitation as a simple consequence of our etiological view. But our identification of functions as a type of disposition, where dispositions are full-fledged entities in reality, allows us to reject (2) categorically.

\section{2. 'Function' and 'functioning' in ordinary language}

A second objection, now specifically aimed at the BFO identification of functions as special sorts of dispositions, reads as follows. Ordinary language draws a distinction between dispositions (continuants) and disposition-realizing processes (occurrents). Thus, there is the disposition: being soluble and there is the corresponding process of realization: dissolving. If functions are dispositions, then we would expect there to be similar term pairs in ordinary language. If the functioning of the heart is the process indicated by the verb 'to pump', then what is the correlated English nominal expression for the realizable attribute or feature? Our response to this is two-fold.

First, while we do give some weight to considerations based on ordinary language when formulating our ontological views, we hold that such considerations are of little force when making decisions as to the correctness or incorrectness of these views themselves. Our reasons for holding that functions are realizable entities turn on ontological arguments to the effect that what is functional exists both as features of bearers and in the realization of those features in processes of functioning.

Second, while the function verb 'to pump' does not have an immediately correlated feature or attribute term (analogous to 'solubility' for 'to dissolve' in English), we note that the disposition to pump is a complex one, resting on a constellation of sub-dispositions for some of which there do indeed exist English terms such as 'ductility,' 'elasticity,' 'resistance,' and so on. Thus we are not overly worried by the lack of English nominal complements for verbs such as 'to pump' or 'to provide oxygen'.

\subsection{Processes and parsimony}

The third objection is that BFO's treatment of functions as dispositions with a history is redundant. Since functions are characteristically realized in processes, would it not be more parsimonious to define functions entirely in terms of processes? To this objection we reiterate the point already made above, namely that there are many entities that clearly do have functions that are never realized, even when the entire lifespan of the entity in question is considered. Even in simple cases such as a knife or a hand there is a significant sense in which they have functions even at times when these functions are in no way being realized. Thus, while acknowledging that what is most conspicuous about functions is their realization in functionings, we insist also that a full account of function that does justice to the facts cannot coherently restrict itself to viewing functions only in terms of the ontological category of processes. Some grounding in continuant features of the relevant entities is necessary, and we have already provided reasons for thinking that dispositions are the best candidates to serve this purpose.

\section{Normativity and malfunctioning}

Röhl and Jansen (2014) have recently offered two further arguments directed specifically against the thesis that a function is (a special subcategory of) disposition, a critique that is embedded in a larger discussion of the recent literature on the ontology of functions. ${ }^{23}$

\footnotetext{
${ }^{23}$ Their critique is based on the elucidation of function in the BFO 2.0 Specification by Smith et al. (2015) discussed above rather than on the more recent formulation in by Arp et al. (2015). While we think the latter is more congenial to some of Röhl
} 


\subsection{The context-dependence objection}

The first turns on the fact that function ascriptions seem to be context dependent in a way that disposition ascriptions are not (Röhl and Jansen, 2014, p. 12). As they point out, a pair of chopsticks available for use in a restaurant and a physically exactly similar pair of sticks lying in the woods may have all their dispositions in common, yet the first has the function of being an eating utensil while the second does not.

Röhl and Jansen describe this case as illustrating a "central difference" between functions and dispositions. If this case is to provide an argument against the BFO thesis that functions are dispositions, then we can imagine two ways in which such an argument might be formulated (see Table 1 below).

Both of these arguments fail, however, for the BFO elucidation of function says only that all functions are dispositions. It does not say that all dispositions are functions. This means that just because two entities have all their dispositions in common, it does not follow that they also have all their functions in common (as was explained in some detail in Section 5.2.1 above). An artifact function is (i) a disposition associated with (ii) a particular historical development of intentional selection or design - and the chopsticks have, while the sticks in the woods lack, a historical development of the needed sort. We thus reject the first premise (P1) of Argument 1. In Argument 2, for reasons discussed in Section 5.2.1 above, we reject the first premise $\left(\mathrm{P}^{\prime}\right)$. The sticks in the woods do not gain a function in the BFO sense, but rather take on what BFO calls a role. The chopstick role is optional for the sticks - its loss will in no way affect their physical structure. ${ }^{24}$

\subsection{The malfunction objection}

Röhl and Jansen's second challenge to the thesis that functions are a kind of disposition turns on the question of whether functions depend for their existence on dispositions. One thing existentially depends on another just in case the first cannot exist without the second. Thus to say that John's height depends upon John is to say that if John did not exist his height would not exist either. On the other hand, a shape's being rectangular does not depend on its being a square, since it is possible for a rectangle to exist that is not a square. According to the BFO elucidation of function as given in Section 4.1 above, however, functions are not dependent on but rather identical to certain dispositions. It is thus not the thesis that functions existentially depend on dispositions that underlies the BFO elucidation of function, yet it is

Table 1

Two formulations of the chopsticks objection

\begin{tabular}{|c|c|}
\hline Chopsticks Argument 1 & Chopsticks Argument 2 \\
\hline $\begin{array}{l}\text { P1. If functions are dispositions, then two entities that have } \\
\text { all dispositions in common have all functions in common as } \\
\text { well. } \\
\text { P2. This is not true for the chopsticks and the sticks in the } \\
\text { above example. } \\
\text { C. It is not the case that functions are dispositions. }\end{array}$ & $\begin{array}{l}\mathrm{P}^{\prime} . \text { When the sticks described above are selected for use as } \\
\text { chopsticks and moved to a restaurant they gain a function. } \\
\mathrm{P} 2^{\prime} \text {. If functions are dispositions, then to gain a function is } \\
\text { always to gain at least one new disposition. } \\
\mathrm{P} 3^{\prime} . \text { Mere change of context cannot change the dispositions } \\
\text { that a thing has. } \\
\mathrm{C}^{\prime} . \text { Functions are not dispositions. }\end{array}$ \\
\hline
\end{tabular}

and Jansen's concerns, it does retain the same core commitments against which their criticisms are addressed, in particular the thesis that functions are dispositions.

${ }^{24}$ As noted in Section 5.2.1, for further discussion of this point, see our response to Artiga's introduction to this special issue of Applied Ontology, which is appended at the end of this paper. 
this latter thesis that Röhl and Jansen strive to argue against. We can ignore this point here, however, since on either reading it should still never be the case that a function exists (a thing "has a function") while the corresponding disposition is lacking.

Röhl and Jansen, however, think that malfunctioning provides precisely such a case, as they purport to show through the example of a carcinomatous lung (Röhl and Jansen, 2014, p. 13). The normal function of a lung is to provide oxygen to the body. When a lung is functioning normally, it has a disposition (or collection of dispositions) that allow it to do this. In the case of a carcinomatous lung, however, there will come a time where these dispositions no longer exist. Yet even then, Röhl and Jansen argue, it is still the function of the lung to provide oxygen to the body. This, therefore, is for them a case where a function exists (the lung "has the function" to do such and such) but the underlying dispositions do not. Thus, they argue, the BFO elucidation of function must be rejected.

This elucidation however conforms to Röhl and Jansen's criterion of SUPPORT, which requires that functions be grounded in the physical structure of their bearers. It also conforms to NORMATIVITY through the fact that this physical structure (and so the associated dispositions) is the result of an intentional design or evolutionary process through which an entity came into (or is maintained in) existence in order to realize processes of a certain sort. It thus seems to get a lot right even by Röhl and Jansen's criteria. So, how much of a challenge is the phenomenon of malfunctioning to this elucidation?

First, we note that functions are often realized along various dimensions to different degrees. Our legs have the function to walk (or to serve walking), but sometimes they serve walking more or less quickly, more or less surely, and so forth. Even where - as for example in the case of the human heart and its function of pumping blood - there is an optimal range of functioning, it is still possible for a heart to be functioning more or less well, including functioning outside of this range, at different times and along different dimensions (for example as concerns regularity of pumping, amount of blood being pumped, efficiency of pumping, rate of pumping, and so forth), and even (in case of transplantation) in different organisms.

The same applies to dispositions in general: a disposition to pump could be at one time a disposition to pump at different rates and with different degrees of vigor at different times. In each of these cases, however, it is still a disposition to pump. Most cases of malfunction do not involve a total annihilation of function, but only non-optimal functioning. There is no challenge to the identification of function as a subtype of disposition here. Indeed, the results of malfunctioning are just what one would expect if functions were a kind of disposition: diminution of functioning is accompanied by a change in enduring underlying disposition.

The only remaining candidate problem case arises when functioning completely and irreversibly stops - for instance when the heart ceases to function entirely and the patient dies or when the heart has been so severely damaged that the patient needs to be kept alive by means of some artificial heart.

\subsubsection{Total malfunction}

For total malfunction to cause problems for the BFO elucidation of function, the dispositions that normally underlie the malfunction in question would need to have been completely destroyed, but in such a way that the associated function can still rightly be said to exist. A case of this sort would be one in which a function exists in the absence of any disposition with which it could be identified. We will thus understand total or complete malfunction to mean just this: a case where the entity in question has been physically damaged to the point where it no longer instantiates to any degree the dispositions normally involved in its functioning.

Can there be a case of this sort where it still seems right to say that the bearer has the associated function? Consider for example a case where the carcinoma in the lungs of a patient is so severe that the 
lungs are incapable of providing oxygen to the body, yet the lungs (in some residual form) survive, and the patient survives because he is still being kept alive by some artificial means. The relevant bearer, the lungs, have completely lost the dispositions that normally underlie their functioning: these dispositions no longer exist.

What, in such a case, is the correct story to tell about the function of the bearer? There seem to us to be three salient ways of understanding such an entity's relation to its function:

Option (1) is the view of Röhl and Jansen, according to which there still survives an instance of the dependent particular function in such a case even when the disposition that would normally make the realization of this function possible is completely absent. According to Röhl and Jansen, this function is (i) essential to its bearer (and so is had by its bearer at any time when the bearer exists at all) and yet (ii) externally grounded (so grounded in or dependent upon something other than the hitherto functionally salient physical structures of the bearer). This then would provide a counterexample to the BFO thesis that functions are a subtype of dispositions.

However, there are at least two further options for describing the case in hand, both of which provide ways of understanding what happens when the dispositions underlying a function cease to exist which do not posit the continued existence of an instance of function as a feature of the bearer under consideration (in this case the lung).

Option (2) says that in a case such as this there is no longer an instance of either disposition or function that inheres in the bearer in question. Both have been destroyed. What is the case is that the lungs in question are supposed to have the instance-level function of providing oxygen to the body but in fact they lack this function. ${ }^{25}$ What grounds this supposed to is the kind of thing that the bearer is, in this case: an instance of the kind lung. On this view being an instance of a kind (such as lung, hand, hammer, and so on) has normative entailments. Instances of a kind are supposed to have features canonical for things of that kind, in particular the functions and dispositions centrally characteristic of the kind. A given instance of a kind may, due to damage, lose these functions and dispositions, but insofar as it still is an instance of that kind, it remains true of it that it is supposed to have them. A key assumption of this view is that historical features and other properties of a completely malfunctioning lung are still sufficient to qualify it as an instance of the kind lung. We note in passing that some form of this assumption is shared also by Röhl and Jansen.

Option (3) also says that in a case such as this there is no longer an instance of either disposition or function that inheres in the bearer in question. Both have been destroyed. However, this view denies that a completely and irreversibly malfunctioning lung can count as an instance of the kind lung. A relatively natural line of reasoning suggests that once an instance of a functional kind such as lung has been rendered completely unable to function as a lung, that entity is no longer an instance of the kind in question. ${ }^{26}$ This view amounts to a rejection of the premise upon which Röhl and Jansen's objection is ultimately based, that a completely malfunctioning lung is still a lung. Options (2) and (3) both represent responses to the argument from complete malfunction that are consistent with the BFO thesis that functions are dispositions.

\subsubsection{Kinds and the definition of function}

While reference to kinds or universals is not explicit in our elucidation of function, it bears repeating that the BFO framework has a built-in commitment to the distinction between universals and particulars.

\footnotetext{
${ }^{25}$ Compare the formal treatment of the lacks relation by Ceusters et al. (2007). See also the discussion in Hoehndorf et al. (2010).

${ }^{26}$ Compare Aristotle, On the Soul, 412 b17 (Aristotle, 1985): An eye which cannot see "is no longer an eye, except in name, just like an eye in stone or a painted eye."
} 
For BFO, functions are in every case functions of certain bearers (material entities), and if a function of a given kind exists, then its bearer must instantiate some independent continuant biological or artifactual universal. Moreover, fully specifying those universals whose instances bear functions - for example placentas or carburetors - will require identifying the function that things of that kind perform. For example, fully describing what lungs are will require identifying the characteristic functions of drawing in oxygen, transmitting it to the bloodstream, exhaling waste gases, and so forth. Fully describing the function of the lung will moreover require a description of the kinds of contexts in which lungs function for example as parts of a human body and in an environment satisfying certain basic conditions regarding atmospheric pressure and so forth. In light of this we take reference to kinds to be unavoidable in almost all talk of functions and take the question of whether or not a given thing has a function to be, at least in part, a question of the kind of thing it is. ${ }^{27}$

\subsection{Advantages of the BFO view of function}

Clearly, therefore, BFO has no problem in withstanding Röhl and Jansen's criticism. But are there reasons to prefer the BFO view over the alternative view which they propose, to the effect that function and disposition are disjoint categories? We can think of at least two. As discussed above, a virtue of the BFO view to the effect that functions are dispositions is that it provides a clear way of understanding the sense in which the function of a thing is a non-optional feature of that thing. While Röhl and Jansen share the BFO view of functions as non-optional, their rejection of the view that functions are tied to the physical structures of their bearers leaves it unclear exactly what makes them non-optional (or "essential," in their idiolect) without appeal to social constructionism or some other variant of epiphenomenalism. Another virtue of the BFO view is that it can account for the causal efficacy of function instances. When an object has a certain function, that function has a causal impact on the world through its identity with a certain disposition instance that is realized under appropriate circumstances. Conversely, when an object does not realize its function (for example because it is completely malfunctioning in the strong sense discussed above) this implies that something about the physical structure of the individual, specifically some of its dispositions, has itself changed or been lost, and this explains its lack of causal efficacy. This is an advantage that the view of Röhl and Jansen lacks, since they are committed to positing instances of function even in cases where these instances make no causal difference whatsoever.

Conclusion. BFO is designed as a top-level domain-neutral ontology that can support the consistent computational representation of empirical data of a maximally broad range of different types. Its success in this regard rests at least in part on its methodology of ontological realism, whereby each of the categories acknowledged by BFO are defended as categories which exist (have instances) in reality. Thus BFO encapsulates the view that there are instances of the kind function in reality, and that the function of your heart to pump blood is one such instance. In the foregoing we have shown how thinking about

\footnotetext{
${ }^{27}$ The significance of associated kinds and contexts for understanding function is explicitly acknowledged in the treatment of function in the recent BFO guidebook (Arp et al., 2015), which elaborates on (Smith et al., 2015) by adding the phrases picked out in bold in what follows:

A function is a special kind of disposition. It is a realizable entity whose realization is an end-directed activity of its bearer that occurs because this bearer is (a) of a specific kind and (b) in the kind or kinds of contexts that it is made or selected for. Thus a function is a disposition that exists in virtue of the bearer's physical make-up, and this physical make-up is something the bearer possesses because of how it came into being - either through natural selection (in the case of biological entities) or through intentional design (in the case of artifacts). Roughly, the entities in question came into being in order to perform activities of a certain sort, called "functionings" (Arp et al., 2015, p. 102).
} 
the issues surrounding the ontology of function in the BFO framework leads to an etiological view of functions as dispositions and have articulated some of the implications of such a view. We believe that we have shown satisfactorily that the BFO account of function can respond to a number of objections to etiological accounts, and we believe that it is robust enough to deal with a range of related criticisms of etiological accounts of function that have been put forward in the literature.

The bearer of a function for BFO is in every case an independent continuant. It is not an objection to BFO that 'function' is sometimes used in other ways, for example to refer to functional processes (when, for example, we smash two randomly selected stones together to light a fire). This is a simple consequence of the fact that BFO is a controlled vocabulary that is designed to provide a regimentation of the way in which general categories are represented that will support interoperability of the domain ontologies defined in its terms. If, on the other hand, we were confronted with a case where we needed to address data concerning such putatively functional processes of a sort which could not be accommodated on the basis of the existing set of BFO categories, then we would consider modifying BFO accordingly in reflection of our commitment to fallibilism.

A related concern turns on BFO's treatment of persons and roles. President Obama the office-holder and Barack Obama the organism are for BFO one and the same entity. In its treatment of functions BFO assumes further that - leaving aside certain artifactual examples such as bacteria specifically cultured to address a certain purpose - only proper parts of organisms can have functions in the BFO sense. How then can BFO deal with what are called the "powers and functions" of the President? How are we to deal with office-holders and organizational entities more generally, where it is not tempting to hold that the putative functions involved would be possessed by their bearers in virtue of the latter's physical structure? BFO has been applied thus far almost always in support of ontologies in scientific domains such as biomedicine or chemistry. Now, however, as BFO is applied in areas such as organizational management and also in intelligence, defense and security, issues relating to the obligations and powers of office-holders are being addressed (Smith et al., 2013).

\section{Acknowledgements}

We thank Adrien Barton, Randall Dipert, Ludger Jansen, Neil Williams, and three anonymous referees for helpful comments and criticisms. We would also like to thank Marc Artiga for his introduction and critical comments on the paper, and Alan Ruttenberg for many valuable contributions.

\section{Appendix. Response to Artiga}

In the foregoing we have developed the BFO view of functions as dispositions whose bearers have a certain type of etiology. Specifically, according to BFO 2.0, $f$ is a function means:

(1) $f$ is a disposition,

(2) $f$ exists in virtue of its bearer's physical make-up

and

(3) this physical make-up is something that this bearer possesses because it came into being, either through evolution (in the case of natural biological entities) or through intentional design (in the case of artifacts), in order to realize processes of a certain sort (Smith et al., 2015). 
We claim that this elucidation satisfies Artiga's desiderata of NORMATIVITY, ACCIDENT, and TELEOLOGY, but hold that Artiga and others are ultimately mistaken in applying to functions the criterion of EPIPHENOMENALISM. Artiga, in his introduction to this special issue, presses us on two main issues, which we deal with in turn.

Total malfunction. The first concerns what we say concerning what makes function ascriptions true in the case of entities that are completely malfunctioning in such a way that the relevant dispositions to function have been destroyed. The specific challenge raised by Artiga is not that our solution is unsuccessful, but rather that it tacitly concedes that functions are not really or not essentially dispositions after all, making our view indistinguishable from standard etiological accounts, for which the truthmaker for $x$ has function $f$ when $x$ is completely malfunctioning would be just the relevant etiology or history of $x$ or of $x \mathrm{~s}$. He wants to conclude from this that we must admit "a sort of normativity that exclusively depends on history and which is possessed by any item irrespective of its actual physical make-up" (Artiga, 2011, pp. 8-9).

Focusing on Artiga's conclusion in the case of totally or completely malfunctioning entities (where relevant dispositions have been completely destroyed) we are tasked with explaining the non-dispositional source of the normativity of functions in a way that still depends on the current physical make-up of the bearer. In responding to Röhl and Jansen's criticisms, we have made it clear that we want to avoid positing a concrete instance of function inhering in a bearer in those cases where the bearer possess no dispositions that could, even in principle, realize that function. The entities we have to work with in providing a response to Artiga are: (i) the remaining physical structure of the entity itself (the lung in our exchange with Röhl and Jansen), (ii) the evolutionary history of that structure as traced through its current possessor's ancestors and its role in their evolutionary success, and (iii) the kind or kinds of thing that the entity is an instance of.

We acknowledge the possibility that a lung might be so physically damaged that it not only completely ceases functioning as a lung but even that it ceases to be a lung at all. We take it that the case where the physical structure of the lung has been completely altered by disease or time such that only a mass of lung cells remain in the chest cavity is no longer a case where we are dealing with a lung properly so called, and so no longer a case where it is true even that the mass of cells is supposed to have the disposition (and so function) to provide oxygen to the body, let alone that it actually has that function.

However, we also acknowledge the possibility of an intermediate state, where the physical structure of the lung has been too incapacitated to continue instantiating the dispositions responsible for its functioning, but not so damaged as to cause the entity in question to no longer instantiate the universal lung. If we call the concrete completely dysfunctional (and so relevant dispositionless) lung $l$, then our view is that it is false to say that $l$ has the function to provide oxygen to the body where this means that there is a concrete functional property instantiated in the lung itself. What we think is true is the assertion that $l$ is supposed to have the function to provide oxygen to the body, which on our view entails that $l$ is supposed to have the relevant disposition involved in providing oxygen to the body. In this case the normative claim is grounded both in the etiology (history) of the entity in question, and in its physical structure, which, though impaired, is still the physical structure of a lung. Against Artiga's suggestion above, the normativity in this case does not depend "exclusively" on the history, but rather both on the history and on the fact that the entity under consideration still has enough determinate physical structure to count as a lung and so as a descendant of past lungs whose possessing certain additional structures and dispositions played a role in the evolutionary success of the organisms of which they were parts. We thus have a non-dispositional source of normativity that depends, at least in part, on the physical makeup of the entity in question, and so - against the conclusion drawn by Artiga, no need "to add a 
non-dispositional category for a property that gives rise to normativity and which is independent of the bearer's current physical make-up" (p. 9).

Naturefacts, repurposed artifacts, and novelty. The second challenge Artiga raises is connected with the possibility of artifactual functions that come into existence solely as the result of intentionally selecting a natural object (what Artiga also calls a "naturefact") or repurposing an already existing artifact. An example of these would be the sticks taken from the woods and intentionally used as chopsticks (where the physical properties of the sticks are assumed to already coincide perfectly with those necessary for chopsticks) already proposed by Röhl and Jansen and discussed by us in Section 7.1. Artiga points out that examples such as these do not sit well with the requirement that the relevant physical structure underlying the function "came into being" in order to realize processes of certain sorts. Here Artiga's challenge can be read as a direct challenge to our definition: there are examples of artifact functions that the definition does not recognize so it is extensionally inadequate.

Artiga's objection can be understood as turning the chopsticks example to a different end: the sticks from the woods are found by someone, recognized as dispositionally suited for use as chopsticks, and then intentionally selected for this purpose. As Artiga points out, one of BFO's criteria for a disposition to count as a function is that the physical make-up that instantiates it must be "something that [its] bearer possesses because it came into being ... in order to realize processes of a certain sort". "Came into being" here strongly suggests that a functional disposition is one whose existence helps to causally explain the existence of the entity, or at least of the physical structure, that it is a disposition of, which is what Artiga's TELEOLOGY desideratum for function requires. Indeed, read in this way our elucidation makes satisfying the teleology desideratum constitutive of something's counting as a function at all. This is a problem, however, since the dispositions (and instantiating physical structures) of the sticks in the woods did not "come into being" in order to realize any particular processes. They are not the result of natural selection, and they pre-exist the human intention to utilize them as chopsticks. If this is right, then the class of entities that Artiga calls "naturefacts", as well as repurposed artifacts (anvils intentionally used as doorstops) pose a challenge to our elucidation. Sections 5.2.1 and 7.1 above discuss this issue to some extent. Our considered view is that, when sticks from the woods are merely intentionally selected to be used as chopsticks, then they do not take on or have a function at all in the BFO sense, but rather a role.

Artiga suggests that satisfying the desideratum of TELEOLOGY according to which the function of a thing helps to explain the existence of that thing seems to come into conflict with satisfying an additional desideratum, which he calls the desideratum of NOVELTY. This requires that an account of artifactual function be able to handle innovative or atypical artifacts such as the chopsticks taken from the woods. Indeed, Artiga goes so far as to suggest that there may be a fundamental conflict in theories of function, such that any theory that satisfies teleology will fail to satisfy novelty, and conversely. If such a fundamental conflict really does exist then it would be as good a sign as any that there are two distinct categories of thing under investigation. The etiological account of functions as dispositions that we are offering here is able to account for both biological and artifactual functions, so long as the functions in question satisfy the TELEOLOGY desideratum. In the biological case, it seems unproblematic to assume that the physical structures and dispositions currently realizing a function in an organism came into being as a result of evolutionary forces related to the survival and reproduction of organisms of the relevant kind. In the artifactual case, the physical structures and dispositions currently realizing a function in an artifact can be seen as coming into being causally as a result of intentional design and manipulation: any artifact whose status and functioning as an artifact depends on physical manipulation or reorganization of its parts conforms to this desideratum. In light of these considerations our view is 
that apparent cases of functioning that fail to conform to the TELEOLOGY desideratum do not involve functions at all, but rather roles (and concomitant dispositions) of the entities in question.

We provided a statement of the current BFO 2.0 elucidation of role in Section 4 above. When a thing has a role it may, in virtue of this fact take on certain powers, duties, or obligations; it may acquire new dispositions to support the sorts of activities associated with the role. But roles do not satisfy anything like Artiga's TELEOLOGY desideratum: the fact that a man is a lawyer in no way explains why he exists, and the fact that mitochondria play a certain role in an experiment equally does not explain why they exist; yet the roles these entities have in those contexts will make them appear to have functions of various sorts. The same could be said of naturefacts and of (merely intentionally) repurposed artifacts generally. These things do not have functions in the BFO sense; but rather roles which, precisely because they do not play any part in explaining the existence of the entities that bear them, are entities of a different kind.

We are thus able to embrace our etiological view of functions as dispositions and thereby satisfy the criteria of TELEOLOGY, NORMATIVITY, and ACCIDENT, while identifying a separate family of entities, namely entities playing certain roles, which are able to satisfy the demands of NOVELTY. In this way we resolve the apparent impasse in theories of function between those that satisfy teleology, normativity and accident on the one hand, and those that satisfy novelty on the other. At the same time we defend ourselves against Artiga's criticism to the effect that BFO falls short of providing a unified elucidation of function of a sort that is applicable to both biological and artifactual functions. This objection would gain a toehold only if chopsticks and naturefacts in general must be assigned to the category of function on our account. However, as we have just argued, this is not the case.

\section{References}

Amundson, R. \& Lauder, G.V. (1994). Function without purpose: The uses of causal role function in evolutionary biology. Biology and Philosophy, 9, 443-469. doi:10.1007/BF00850375.

Aristotle (1985). The Complete Works of Aristotle (Vol. 2). J. Barnes (Ed.). Oxford: Oxford University Press.

Arp, R., Smith, B. \& Spear, A. (2015). Building Ontologies with Basic Formal Ontology. Cambridge, MA: MIT Press.

Artiga, M. (2011). Re-organizing organizational accounts of function. Applied Ontology, 6, 105-124.

Ashburner, M., Ball, C.A., Blake, J.A., Botstein, D., Butler, H., Cherry, J.M., Davis, A.P., Dolinski, K., Dwight, S.S., Eppig, J.T., Harris, M.A., Hill, D.P., Issel-Tarver, L., Kasarskis, A., Lewis, S., Matese, J.C., Richardson, J.E., Ringwald, M., Rubin, G.M. \& Sherlock, G. (2000). Gene ontology: Tool for the unification of biology. Nature Genetics, 25(1), 25-29. doi:10.1038/75556.

Berg, J.M., Tymoczko, J.L. \& Styer, L. (2002). Biochemistry (5th ed.). New York: W H Freeman.

Bigelow, J. \& Pargetter, R. (1987). Functions. Journal of Philosophy, 84, 181-196. doi:10.2307/2027157.

Buller, D. (Ed.) (1999). Function, Selection, and Design Albany. NY: SUNY Press.

Buller, D.J. (1998). Etiological theories of function: A geographical survey. Biology and Philosophy, 13, 505-527. doi:10.1023/ A: 1006559512367.

Ceusters, W., Elkin, P. \& Smith, B. (2007). Negative findings in Electronic Health Records and biomedical ontologies: A realist approach. International Journal of Medical Informatics, 76, 326-333. doi:10.1016/j.jimedinf.2007.02.003.

Cummins, R. (1975). Functional analysis. Journal of Philosophy, 72, 741-765. doi:10.2307/2024640.

Díaz-Herrera, P. (2006). What is a biological function? In Proceedings of FOIS 2006 (pp. 128-140).

Dipert, R. (1993). Artifacts, Art Works, and Agency. Philadelphia: Temple University Press.

Ellis, B. \& Liese, C. (1994). Dispositional essentialism. Australasian Journal of Philosophy, 72(1), 27-45. doi:10.1080/ 00048409412345861.

Fara, M. (2005). Dispositions and habituals. Noûs, 39, 43-82.

Gilson, E. (1984). From Aristotle to Darwin and Back Again: A Journey in Final Causality, Species, and Evolution. San Francisco: Ignatius Press.

Goldfain, A., Smith, B. \& Cowell, L.G. (2011). Towards an ontological representation of resistance: The case of MRSA. Journal of Biomedical Informatics (Feb.), 44(1), 35-41. doi:10.1016/j.jbi.2010.02.008. 
Handfield, T. (Ed.) (2009). Dispositions and Causes. Oxford: Oxford University Press.

Hoehndorf, R., Ngomo, A.-C.N. \& Kelso, J. (2010). Applying the functional abnormality ontology pattern to anatomical functions. Journal of Biomedical Semantics, 1, 4. doi:10.1186/2041-1480-1-4.

Krohs, U. \& Kroes, P. (2009). Functions in Biological and Artificial Worlds: Comparative Philosophical Perspectives. Cambridge, MA: MIT Press.

Laurin, M., Everett, M.L. \& Parker, W. (2011). The cecal appendix: One more immune component with a function disturbed by post-industrial culture. The Anatomical Record, 294(4), 567-579. doi:10.1002/ar.21357.

Lewis, D. (1997). Finkish dispositions. The Philosophical Quarterly, 47, 143-158. doi:10.1111/1467-9213.00052.

Leunissen, M. (2010). Explanation and Teleology in Aristotle's Science of Nature. Cambridge: Cambridge University Press.

Lowe, E.J. (2006). The Four-Category Ontology. A Metaphysical Foundation for Natural Science. Oxford: Clarendon Press.

Lowe, E.J. (2010). On the individuation of powers. In A. Marmodoro (Ed.), The Metaphysics of Powers: Their Grounding and Their Manifestations (pp. 8-26). New York: Routledge.

Millikan, R. (1984). Language, Thought, and Other Biological Categories. Cambridge: MIT Press.

Mizoguchi, R., Kitamura, Y. \& Borgo, S. (2012). Towards a unified definition of function. In M. Donnelly and G. Guizzardi (Eds.), Formal Ontology in Information Systems (FOIS 2012) (pp. 103-116). Amsterdam: IOS Press.

Mossio, M., Saborido, C. \& Moreno, A. (2009). An organisational account of biological functions. British Journal for Philosophy of Science, 60, 813-841. doi:10.1093/bjps/axp036.

Mumford, S. (1996). Conditionals, functional essences, and Martin on dispositions. The Philosophical Quarterly, 46(182), 86-92. doi:10.2307/2956310.

Röhl, J. \& Jansen, L. (2014). Why functions are not special dispositions: An improved classification of realizables for top-level ontologies. Journal of Biomedical Semantics, 5, 27. doi:10.1186/2041-1480-5-27.

Rosse, C. \& Mejino, J.L.V. (2003). A reference ontology for bioinformatics: The foundational model of anatomy. Journal of Biomedical Informatics, 36, 478-500. doi:10.1016/j.jbi.2003.11.007.

Searle, J. (1995). The Construction of Social Reality. New York: Free Press.

Smith, B. (2005). Against fantology. In J.C. Marek and M.E. Reicher (Eds.), Experience and Analysis (pp. 153-170). Vienna: HPT\&ÖBV.

Smith, B. (2012). Classifying processes: An essay in applied ontology. Ratio, 25(4), 463-488. doi:10.1111/ j.1467-9329.2012.00557.x

Smith, B., et al. (2015). Basic Formal Ontology 2.0: Specification and user's guide. Available at: https://github.com/ BFO-ontology/BFO, accessed December 14, 2015.

Smith, B., Malyuta, T., Rudnicki, R., Mandrick, W., Salmen, D., Morosoff, P., Duff, D.K., Schoening, J. \& Parent, K. (2013). IAO-Intel: An Ontology of Information Artifacts in the Intelligence Domain. In K.B. Laskey and P.C.G. Costa (Eds.), Proceedings of the Eighth International Conference on Semantic Technologies for Intelligence, Defense, and Security (STIDS 2013), Fairfax, VA, CEUR Workshop Proceedings (Vol. 1097, pp. 33-40).

Smith, B., Papakin, I. \& Munn, K. (2003). Bodily systems and the modular structure of the human body. In Proceedings of the 9th Conference on Artificial Intelligence in Medicine. Lecture Notes on Artificial Intelligence (Vol. 2780, pp. 86-90), Berlin: Springer.

Spear, A.D. (2006). Ontology for the 21st century: An introduction with recommendations. Available at: http://www.ifomis. org/bfo/documents/manual.pdf.

Spear, A.D. \& Smith, B. (2015). Defining ‘function'. In Third International Workshop on Definitions in Ontologies. Available at: https://sites.google.com/site/defsinontos2015/accepted-papers, accessed May 9, 2016.

Wouters, A. (2005). The function debate in philosophy. Acta Biotheoretica, 53, 123-151. doi:10.1007/s10441-005-5353-6. 